IEM-FT-188/99

IFT-UAM/CSIC-99-10

hep-ph/9903274

March 1999

\title{
ELECTROWEAK BARYOGENESIS IN THE MSSM ${ }^{a}$
}

\author{
M. QUIROS, M. SECO \\ Instituto de Estructura de la Materia, \\ E-28006, Madrid, SPAIN \\ e-mail: mariano@makoki.iem.csic.es
}

\begin{abstract}
We review the baryogenesis scenario in the MSSM at the perturbative level and, in particular, the impact of two-loop corrections on the strength of the phase transition and the amount of generated baryon asymmetry. We confirm the baryogenesis window, where $m_{H} \lesssim 115 \mathrm{GeV}$, for $m_{Q} \lesssim$ a few $\mathrm{GeV}$, and the right-handed stop mass is constrained in the region, $100 \mathrm{GeV} \lesssim m_{\tilde{t}} \lesssim m_{t}$. This scenario will be tested at LEP and Tevatron colliders.
\end{abstract}

\section{Introduction}

Electroweak baryogenesis is an appealing mechanism to explain the observed value of the baryon-to-entropy ratio, $n_{B} / s \sim 10^{-10}$, at the electroweak phase transition 2 , that can be tested at present and future high-energy colliders Although the Standard Model (SM) contains all the necessary ingredients 1 for a successful baryogenesis, it fails in providing enpugh baryon asymmetr In particular it has been proven by perturbative $3 \mathrm{H}$ and non-perturbative 6 methods that, for Higgs masses allowed by present LEP bounds, the phase transition js too weakly first order or does not exist at all (it is an analytical cross-over $)$, and any previously generated baryon asymmetry would be washed out after the phase transition. On the other hand the amount of $\mathrm{CP}$ violation arising from the CKM phase is too small for generating the observed baryon asymmetry . Therefore electroweak baryogenesis requires physics beyond the Standard Model at the weak scale.

Among the possible extensions of the Standard Model at the weak scale, its minimal supersymmetric extension (MSSM) is the best motivated one. It provides a technical solution to the hierarchy problem and has deep roots in more fundamental theories unifying gravity with the rest of interactions. As for the

${ }^{a}$ Plenary talk given at Strong and Electroweak Matter (SEW98), 3 December 1998, Copenhagen. 
strength of the phase transition 9-14, a region in the space of supersymmetric parameters has been found $2-20$ where the phase transition is strong enough to let sphaleron interactions go out of equilibrium after the phase transition and not erase the generated baryon asymmetry. This region (the so-called light stop scenario) provides values of the lightest Higgs and stop eigenstate masses which will be covered at LEP2 and Tevatron colliders.

The MSSM has ney violating phases 21 that can drive enough amount of baryon asymmetry $22-28$ provided that the previous phases are not much less than 1 and the charginos and neutralinos are not heavier than $200 \mathrm{GeV}$. In all calculations of the baryon asymmetry the details of the wall parameters play a prominent role in the final result. In particular the wall thickness, $L_{\omega}$, and the relative variation of the two Higgs fields along the wall, $\Delta \beta$, are typical parameters which the generated baryon asymmetry depends upon. Although reasonable assumptions about the Higgs profiles along the wall have been done, as e.g. kinks or sinusoidal patterns interpolating between the broken and the symmetric phases, as well as estimates on the value of $\Delta \beta$ based on purely potential energy considerations, it is clear that the reliability of those estimates as well as more precise computations of the baryon asymmetry should rely on realistic calculations of the Higgs profiles and the tunneling processes from the false to the true vacuum Such a task, achieved in the case of one Higgs field in the Standard Model 29, has been recently done in the case of two-Higgs doublets of the MSSM in Refs. [30, 31, 32].

In this talk we will review the impact of two-loop corrections in the MSSM effective potential both for the strength of the phase transition and for the calculation of the bubble parameters. We will show that they produce an enhancement of both the strength of the phase transition (leading to the possibility of encompassing higher values of the Higgs mass) and of the amount of generated baryon asymmetry (leading to alleviated bounds on the value of the CP-violating parameters).

\section{Phase transition: two-loop enhancement}

The possibility of achieving, in the MSSM, a strong-enough phase transition for not washing out any previously generated baryon asymmetry, characterized by the condition

$$
v\left(T_{c}\right) / T_{c} \gtrsim 1
$$

has been recently strengthened by three facts:

- The presence of light $\widetilde{t}_{R}$ (with small mijipg $\widetilde{A}_{t}$ considerably enhances the strength of the phase transition 10.11.12, [3. This is the so-called light stop scenario. 
- Two-loop correctipgs enhance the phase transition in the SME, and in the MSSML1. 18.192 .20$.

- The validity of perturbation theory, and in particular the results of twoloop calculations, for the light stop scenario has been recently confirmed by non-perturbative results 33 .

In Fig. 1, left panel, we show the two- and one-loop approximation for the order parameter $v\left(T_{c}\right) / T_{c}$ as a function of $m_{A}$ for the values of supersymmetric parameters which are indicated in the caption. We can see that the one-loop approximation does not satisfy condition (II), and the corresponding case would be ruled out, while the two-loop approximation does; two-loop corrections are able to rescue this case.
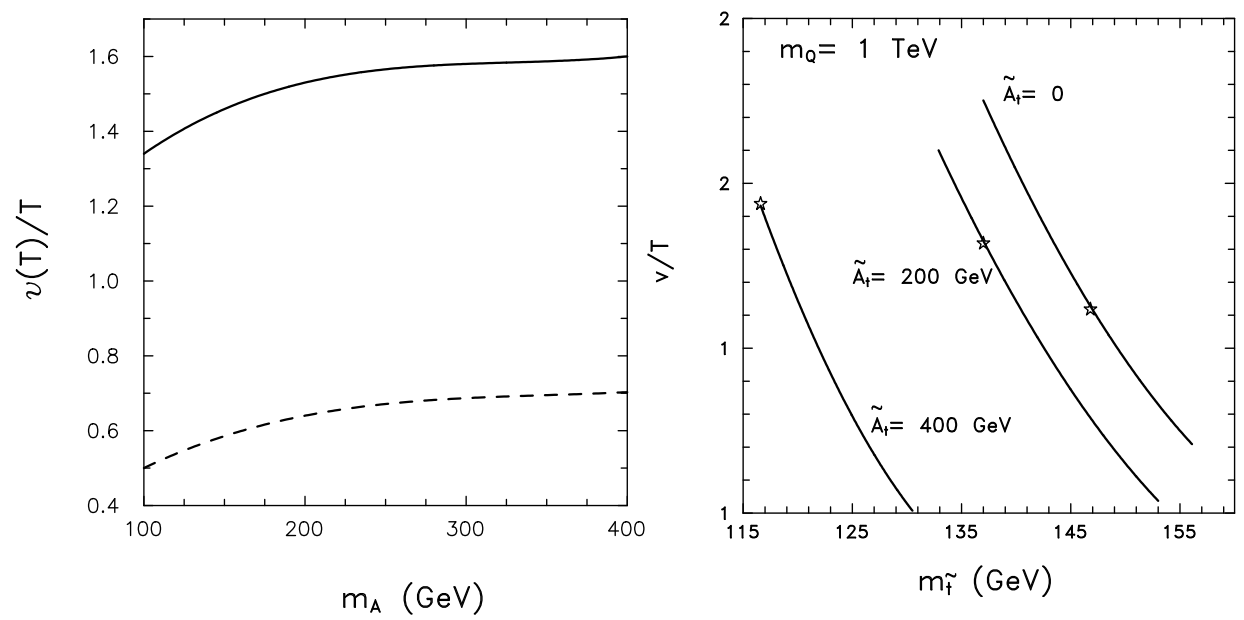

Figure 1: Left panel: $v\left(T_{c}\right) / T_{c}$ as a function of $m_{A}$ in the two-loop (solid) and one-loop (dashed) approximations for $m_{Q}=1 \mathrm{TeV}, \tan \beta=2.5, m_{\widetilde{t}_{R}}=150 \mathrm{GeV}$ and $\widetilde{A}_{t}=0$. Right panel: $v\left(T_{c}\right) / T_{c}$ as a function of $m_{\tilde{t}_{R}}$ for $m_{Q}=1 \mathrm{TeV}, \tan \beta=3$ and various values of the mixing. The value of the Higgs mass varies between $\sim 90$ and $95 \mathrm{GeV}$.

However the price the light stop scenario has to pay is that it may require moderately negative values of the supersymmetric parameter $m_{U}^{2} \equiv-\widetilde{m}_{U}^{2}$ and then, apart from the electroweak minimum along the Higgs $(\phi)$ direction, another color breaking minimum along the $U \equiv \widetilde{t}_{R}$ direction might appear. Therefore both directions should be studied at finite temperature. A preview of our results is shown in Fig. 1, right panel, where $v\left(T_{c}\right) / T_{c}$ is plotted versus 
Table 1: Mass spectrum along the $U$ direction.

\begin{tabular}{|c|c|c|}
\hline field & d.o.f. & mass $^{2}$ \\
\hline 4 gluons & 12 & $g_{s}^{2} U^{2} / 2$ \\
1 gluon & 3 & $2 g_{s}^{2} U^{2} / 3$ \\
$1 B$ gauge boson & 3 & $g^{\prime 2} U^{2} / 9$ \\
5 squark-goldstones & 5 & $m_{U^{2}}^{2}+g_{s}^{2} U^{2} / 3$ \\
1 squark & 1 & $m_{U}^{2}+g_{s}^{2} U^{2}$ \\
\hline 4 $\widetilde{Q}_{L}$-Higgs & 4 & $-m_{H}^{2} / 2+h_{t}^{2} \sin ^{2} \beta U^{2}$ \\
2 Dirac fermions $\left(t_{L}, \widetilde{H}\right)$ & 8 & $\mu^{2}+h_{t}^{2} U^{2}$ \\
\hline
\end{tabular}

$m_{\widetilde{t}_{R}}$ for different values of the mixing parameter. We can see that, as anticipated, $v\left(T_{c}\right) / T_{c}$ increases when the stop-right mass decreases, for all values of $\widetilde{A}_{t}$. However, for given values of $m_{\widetilde{t}_{R}}$, which are indicated with a star in the plot, the electroweak minimum stops being the true minimum at the temperature $T_{c}$ and the phase transition proceeds first toward the color breaking minimum along the $U$ direction. For instance for $\widetilde{A}_{t}=0$ this happens at $m_{\widetilde{t}_{R}}=147 \mathrm{GeV}$, while for $\widetilde{A}_{t}=400 \mathrm{GeV}$ it happens at $m_{\widetilde{t}_{R}}=117 \mathrm{GeV}$. Moreover all lines stop at some value of $m_{\widetilde{t}_{R}}$ where the electroweak minimum becomes unstable.

To systematically analyze the different possibilities we have computed the two-loop effective potential along the $\phi$ and $U$ directions and compared their cosmological evolutions with $T$. The two-loop effective potential along the $\phi$ direction was carefully studied in Refs. [17, 18]. The one-loop correction is dominated by the exchange of the top/stop sector while the two-loop effective potential is given by two-loop diagrams with stops and gluons, as well as one-loop diagrams with the stop thermal counterterm. The two-loop effective potential along the $U$ direction was studied in Refs. [19, 20]. The mass spectrum is given in table 1 , where we have considered small mixing $\widetilde{A}_{t} / m_{Q}$ and large gluino masses. The one-loop diagrams correspond to the propagation of gluons, squarks and Higgses as well as Dirac fermions. The leading two-loop contributions correspond to sunset and figure-eight diagrams with the fields of table 1 propagating, as well as one-loop diagrams with thermal counterterms insertions corresponding to gluons and squarks.

For a given value of the supersymmetric parameters we have computed $T_{c}$, the critical temperature along the $\phi$ direction, and $T_{c}^{U}$, the critical temperature along the $U$ direction. The comparison of them will provide information about the cosmological evolution of the system. We have plotted in Fig. 2 2 the critical 
temperatures as a function of $m_{\widetilde{t}_{R}}$ for the values of the supersymmetric parameters indicated in the caption, and for different values of the mixing parameter $\widetilde{A}_{t}$. For a given point the phase transition will happen first along the direction whose critical temperature is higher. Then for $\widetilde{A}_{t}=0$ the phase transition will proceed along the $\phi(U)$ direction for $m_{\widetilde{t}_{R}} \gtrsim 147 \mathrm{GeV}\left(m_{\widetilde{t}_{R}} \lesssim 147 \mathrm{GeV}\right)$. For $\widetilde{A}_{t}=200 \mathrm{GeV}$ the phase transition will proceed along the $\phi(U)$ direction for $m_{\widetilde{t}_{R}} \gtrsim 137 \mathrm{GeV}\left(m_{\widetilde{t}_{R}} \lesssim 137 \mathrm{GeV}\right)$, and for $\widetilde{A}_{t}=400 \mathrm{GeV}$ the phase transition will proceed always along the $\phi(U)$ direction. These results are in agreement with those of Fig. 11.
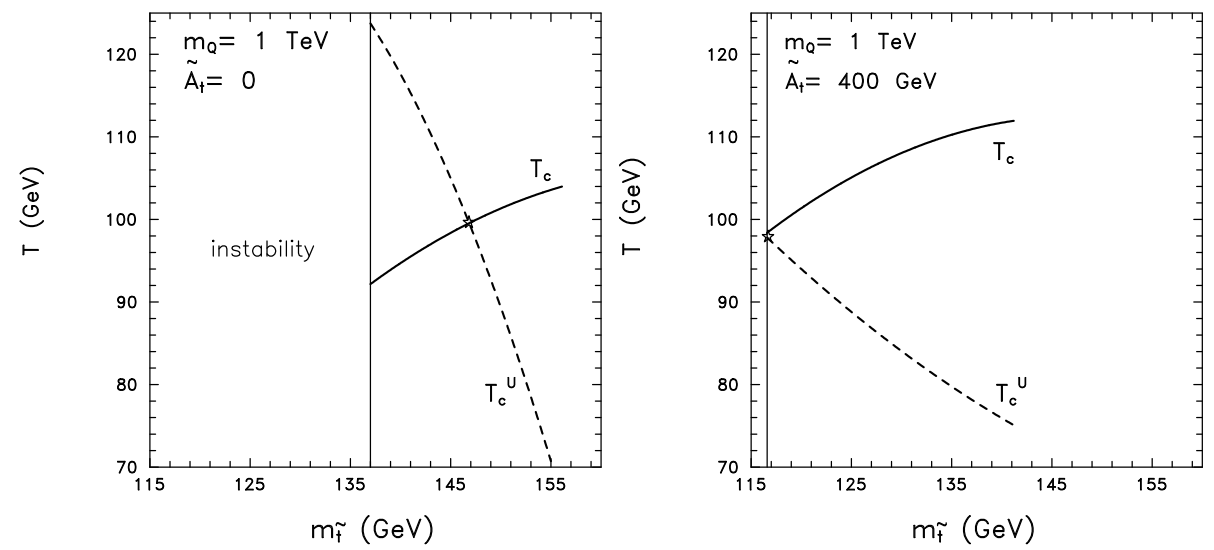

Figure 2: Plot of the critical temperatures $T_{c}$ and $T_{c}^{U}$ for $m_{Q}=1 \mathrm{TeV}, \tan \beta=3$ and: $\widetilde{A}_{t}=0, m_{H}=89 \mathrm{GeV}$, left panel; and, $\widetilde{A_{t}}=400 \mathrm{GeV}, m_{H}=94 \mathrm{GeV}$, right panel.

Therefore, four different situations can arise:

- a) $T_{c}^{U}<T_{c}$ and $\widetilde{m}_{U}<m_{U}^{c} \equiv\left(m_{H}^{2} v^{2} g_{s}^{2} / 12\right)^{1 / 4} 12$. In this case the phase transition proceeds first along the $\phi$ direction and the field remains at the electroweak minimum forever. This region is called stability region.

- b) $T_{c}^{U}<T_{c}$ and $\tilde{m}_{U}>m_{U}^{c}$. In this region the electroweak minimum is metastable (metastability region). It can be physically acceptable provided that its lifetime is larger than the age of the universe at this temperature: $\Gamma_{\phi \rightarrow U}<H^{34}$.

- c) $T_{c}^{U}>T_{c}$ and $\tilde{m}_{U}<m_{U}^{c}$. In this case the $U$-phase transition happens first and therefore it is physically acceptable provided that the lifetime is 
shorter than the age of the universe: $\Gamma_{U \rightarrow \phi}>H 35$. This region is called two step region.

- d) $T_{c}^{U}>T_{c}$ and $\widetilde{m}_{U}>m_{U}^{c}$. This is the region of instability of the electroweak minimum. It is absolutely excluded.
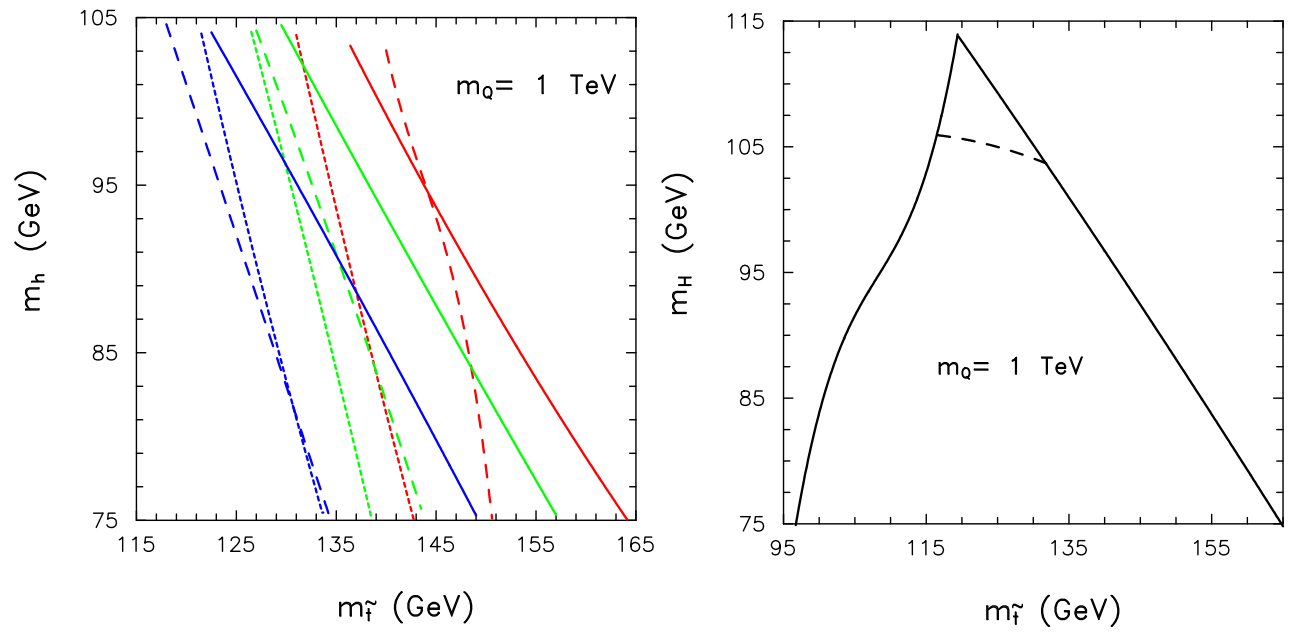

Figure 3: Left panel: values of $m_{h}, m \sim$ for which $v\left(T_{c}\right) / T_{c}=1$ (solid line), $T_{c}^{U}=T_{c}$ (dashed line), $\widetilde{m}_{U}=\widetilde{m}_{U}^{c}$ (short-dashed line), for $m_{Q}=1 \mathrm{TeV}$ and $\widetilde{A}_{t}=0,200,300 \mathrm{GeV}$. The region on the left of the solid line is consistent with a strongly first order phase transition. A two step phase transition may occur in the regions on the left of the dashed line, while on the left of the short-dashed line, the physical vacuum at $T=0$ becomes metastable. The region on the left of both the dashed and short-dashed lines leads to a stable color breaking vacuum state at zero temperature and is hence physically unacceptable. Right panel: The absolute region of stability in the $\left(m_{H}, m_{\tau_{R}}\right)$ plane, for $m_{Q}=1 \mathrm{TeV}$ (below the dashed line) and for higher values of $m_{Q}, \sim$ a few $\mathrm{TeV}$ (the region inside the solid lines).

Fig. 3, left panel, shows the region of parameter space consistent with a sufficiently strong phase transition for $m_{Q}=1 \mathrm{TeV}, \widetilde{A}_{t}=0,200,300 \mathrm{GeV}$. For low values of the mixing, $\widetilde{A}_{t} \lesssim 200 \mathrm{GeV}$, case a) or c) may occur but, contrary to what happens at one-loop, case b) is not realized For the case of no mixing, this result is in agreement with the analysis of 19 . The region of absolute stability of the physical vacuum for $\widetilde{A}_{t} \simeq 0$ is bounded to values of the Higgs mass of order $95 \mathrm{GeV}$. There is a small region at the right of the solid line, in which a two-step phase transition may take place, for values of the parameters which would lead to $v / T<1$ for $T=T_{c}$, but may evolve to larger values at some $T<T_{c}$ at which the second of the two step phase transition into the 
physical vacuum takes place. This region disappears for larger values of the stop mixing mass parameter. For values of the mixing parameter $\widetilde{A}_{t}$ between $200 \mathrm{GeV}$ and $300 \mathrm{GeV}$, both situations, cases b) and c) may occur, depending on the value of $\tan \beta$. For large values of the stop mixing, $\widetilde{A}_{t}>300 \mathrm{GeV}$, a two-step phase transition does not take place. Fig. 3, right panel, shows the absolute region of stability for $m_{Q} \leq 1 \mathrm{TeV}$, below the dashed line, where we can see an absolute upper bound on the Higgs mass $\sim 105 \mathrm{GeV}$. If we relax the condition on $m_{Q}$ and allow for values $\sim 2-3 \mathrm{TeV}$, then we get the bound, corresponding to the region inside the solid lines, $\sim 115 \mathrm{GeV} 36$.

\section{Electroweak baryogenesis: two-loop enhancement}

The $C P$ violating current $\left\langle J_{\mathscr{C}}\right\rangle_{\text {has }}$ been computed using triangle diagrams in a 'Higgs insertion expansion'2225. Two kinds of diagrams are considered: stop mediated and Higgsino-gaugino mediated ones. We find the former negligible due to the fact that we are considering left-handed stops much heavier than the temperature and therefore decoupled from the thermal bath. The latter are found to be dominant and the amount of generated baryon asymmetry is found, after taking for simplicity the thick wall limit, $t_{\omega}=L_{\omega} / v_{\omega} \gg \tau_{\widetilde{t}, \widetilde{H}, \ldots}=$ $1 / \Gamma_{\widetilde{t}, \widetilde{H}, \ldots}$, where $v_{\omega}$ is the wall velocity, as:

$$
\frac{n_{B}}{s} \simeq f\left(m_{Q}, m_{\widetilde{t}}, m_{\widetilde{H}}, \Gamma_{\widetilde{t}}, \Gamma_{\widetilde{H}}, \ldots\right)\left\langle\frac{v^{2}\left(T_{c}\right)}{T_{c}^{2}}\right\rangle \Delta \beta\left(T_{c}\right)
$$

where $\left\langle v^{2}(T)\right\rangle$ is the integral of the Higgs profile along its radial coordinate, $\Delta \beta(T)$ the variation of the angle $\beta(T)$ along the bubble wall and the function $f$ comes from the integral of the Feynman diagrams and integration of the diffusion equations. In this way the main dependence on the phase transition is concentrated on the parameter $\Delta \beta\left(T_{c}\right)$.

We have computed the bubble solutions of the MSSM 30 using, for the sake of comparison, the one- and two-loop effective potential. We have confirmed the goodness of the thick wall approximation $\left(L_{\omega} T_{c} \gg 10 v_{\omega}\right)$ and found, on $n_{B} / s$, a two-loop enhancement, with respect to the one-loop result, which goes between one and two orders of magnitude.

In Fig. 4, left panel, we plot $\Delta \beta(T)$ for the values of the supersymmetric parameters indicated in the figure caption. The solid line is the result of our numerical calculation and the diamond corresponds to the actual value of the critical temperature for the considered case. For the sake of comparison we also plot (dashed line) the result assuming that the bubble solution proceeds along the path of minimal potential energy. We can see that there can be a 

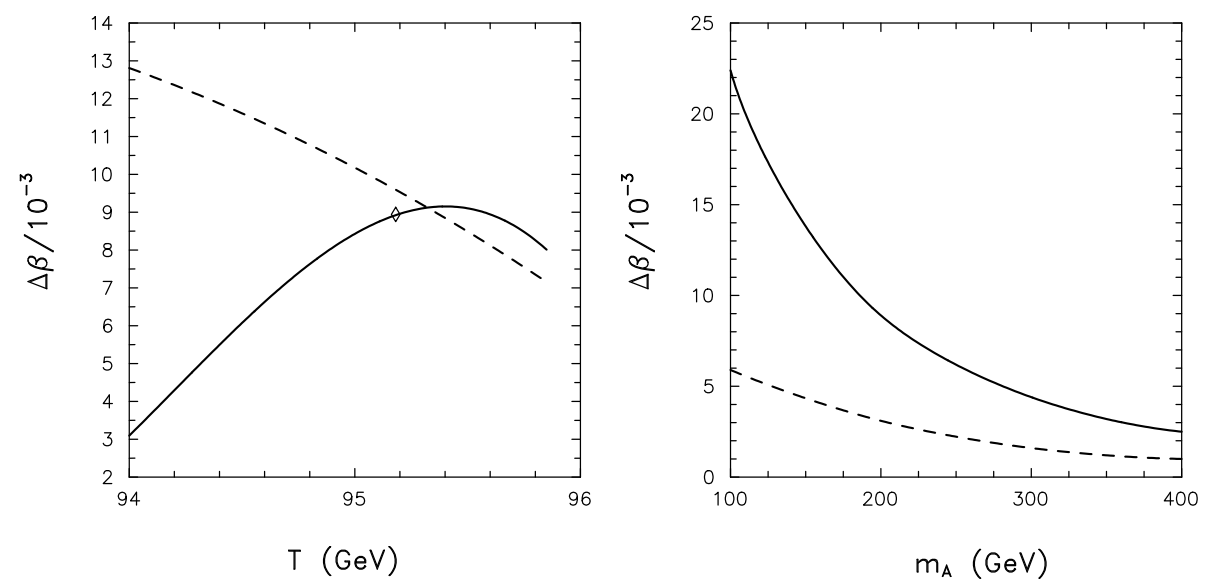

Figure 4: Left panel: the parameter $\Delta \beta$ as a function of the temperature, for $m_{Q}=1 \mathrm{TeV}$, $\tan \beta=2.5, \widetilde{A}_{t}=0, m_{\tilde{t}}=150 \mathrm{GeV}$ and $m_{A}=200 \mathrm{GeV}$, from our numerical calculations (solid curve) and as obtained by pure potential energy considerations (dashed curve). The diamond indicates the value of the critical temperature for the considered case. Right panel: the parameter $\Delta \beta$ in the two-loop (solid curve) and one-loop (dashed curve) approximations for the same values of the supersymmetric parameters.

large deviation between both results, the responsibility being the contribution of the kinetic energy to the total action. In Fig. 1 , right panel, we plot the parameter $\Delta \beta\left(T_{c}\right)$ as a function of $m_{A}$ in the two-loop (solid line) and oneloop (dashed line) approximations. Notice that the value of $T_{c}$, as well as $\Delta \beta$, is different from point to point, for different values of $m_{A}$. We can see that the ratio $\Delta \beta_{2 L} / \Delta \beta_{1 L}$ varies from $\sim 3.6$, for $m_{A}=100 \mathrm{GeV}$, to $\sim 2.5$, for $m_{A}=400 \mathrm{GeV}$. This enhancement, along with the two-loop enhancement that we obtained for the quantity $v^{2} / T^{2}$, that can be quantified from Fig. 11 as $~$ 6.7 for $m_{A}=100$ and $\sim 5.2$ for $m_{A}=400 \mathrm{GeV}$, provides a total enhancement in $n_{B} / s$, see Eq. (2), which can go from $\sim 24$, for $m_{A}=100 \mathrm{GeV}$, to $\sim 13$, for $m_{A}=400 \mathrm{GeV}$.

Since the function $f$ in Eq. 22 goes linear in $\varphi_{\mu}$ (the phase of the complex $\mu$ ), this enhancement translates into a smaller value for $\varphi_{\mu}$ if we fix $n_{B} / s$ to its experimental value. Therefore the value of $\varphi_{\mu}$ in the two-loop approximation is smaller than the corresponding one in the one-loop approximation, for a fixed value of $n_{B} / s$ This is shown in Fig. 5, left panel, which is based on our numerical solutions 30 and shows a two-loop enhancement $\sim 22$ for $m_{A}=100$ $\mathrm{GeV}$, and $\sim 13$ for $m_{A}=400 \mathrm{GeV}$, in agreement with_our previous rough estimates. The corresponding prediction for $\varphi_{\mu}$ from (2) 22 is shown in Fig. 5 , 

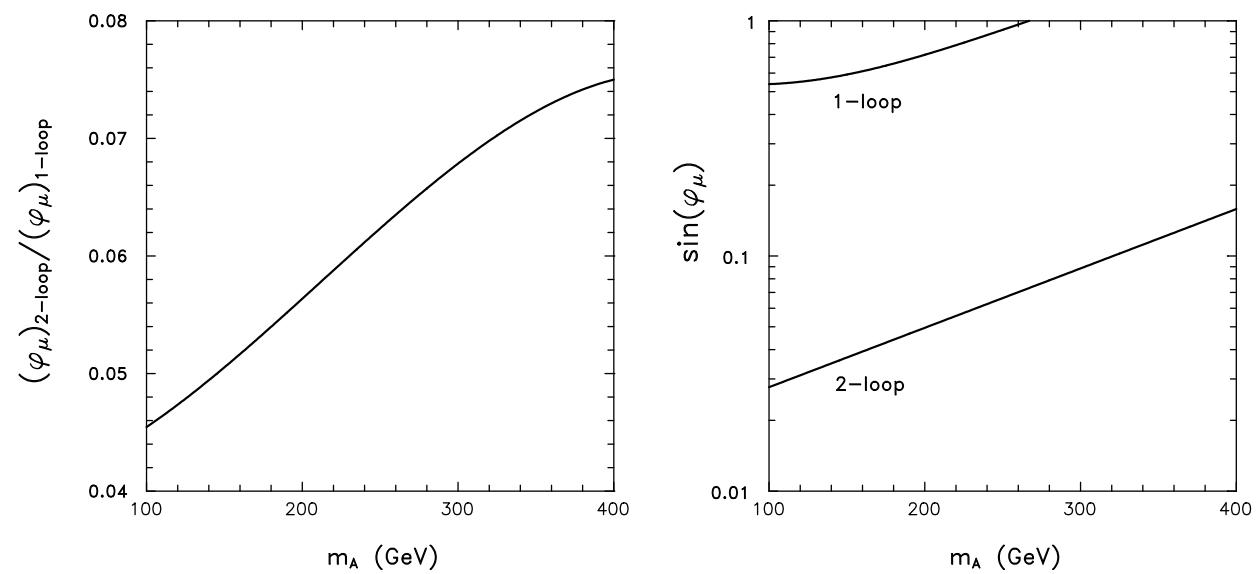

Figure 5: Left panel: the two-to-one loop ratio (two-loop enhancement) of $\varphi_{\mu}$ for the same values of supersymmetric parameters as in Fig. A. Right panel: two- and one-loop calculations of the CP violating parameter $\varphi_{\mu}$ as obtained by fixing $n_{B} / s$ to its experimental value and using the calculation of (2).

right panel where we can see that the $\mathrm{CP}$ violating parameter can be much smaller than that predicted by the one-loop approximation.

\section{Conclusions}

Our results seem to point toward electroweak baryogenesis in the MSSM. There is a baryogenesis window in the $\left(m_{H}, m_{\widetilde{t}}\right)$ plane summarized as:

- The Higgs mass is constrained to $m_{H} \lesssim 105 \mathrm{GeV}$ for $m_{Q} \lesssim 1 \mathrm{TeV}$. This constrain can be relaxed if we allow left-handed stop masses to go to the few $\mathrm{TeV}$ range, in which case the bound is $m_{H} \lesssim 115 \mathrm{GeV}$. We can compare these numbers with the experimental bounds on the Higgs mass at LEP.

- The present LEP bound is $\sim 95 \mathrm{GeV}$.

- At the end of the present run at LEP the bound on $m_{H}$ will increase to $\sim 100 \mathrm{GeV}$.

- The LEP upgrade at $\sqrt{s}=200 \mathrm{GeV}$, in 1999-2000, will explore the Higgs mass up to $\sim 105-110 \mathrm{GeV}$.

As a consequence LEP will cover most of the Higgs window allowed by baryogenesis. 
- The stop mass is bounded to lie in the range $100 \mathrm{GeV} \lesssim m_{\widetilde{t}} \lesssim m_{t}$. The lower bound can be relaxed a bit (to $80 \mathrm{GeV}$ ) if metastability solutions are satisfactory 34 . A wide region of stop masses will be tested at the next Tevatron run (starting end 1999). The whole region will be tested at TeV33.

To conclude, baryogenesis at the MSSM seems to be viable and provides a very specific window in the space of supersymmetric parameters. This scenario will be tested in the near future by collider experiments.

\section{Acknowledgments}

We warmly thank our collaborators M. Carena, J. Moreno, A. Riotto, I. Vilja and C. Wagner, which whom recent works on the subject have been done. This work is partly supported by CICYT (Spain) under contract AEN98-0816.

\section{References}

1. A.D. Sakharov, JETP Lett. 91B (1967) 24

2. For reviews, see: A.G. Cohen, D.B. Kaplan and A.E. Nelson, Annu. Rev. Nucl. Part. Sci. 43 (1993) 27; M. Quirós, Helv. Phys. Acta 67 (1994) 451; V.A. Rubakov and M.E. Shaposhnikov, Phys. Usp. 39 (1996) 461-502 hep-ph/9603208; M. Quirós, hep-ph/9901312; A. Riotto And M. Trodden hep-ph/9901362

3. G.W. Anderson and L.J. Hall, Phys. Rev. D45 (1992) 2685

4. M.E. Carrington, Phys. Rev. D45 (1992) 2933; M. Dine, R.G. Leigh, P. Huet, A. Linde and D. Linde, Phys. Lett. B283 (1992) 319; Phys. Rev. D46 (1992) 550; P. Arnold, Phys. Rev. D46 (1992) 2628; J.R. Espinosa, M. Quirós and F. Zwirner, Phys. Lett. B314 (1993) 206; W. Buchmüller, Z. Fodor, T. Helbig and D. Walliser, Ann. Phys. 234 (1994) 260

5. J. Bagnasco and M. Dine, Phys. Lett. B303 (1993) 308; P. Arnold and O. Espinosa, Phys. Rev. D47 (1993) 3546; Z. Fodor and A. Hebecker, Nucl. Phys. B432 (1994) 127

6. K. Kajantie, K. Rummukainen and M.E. Shaposhnikov, Nucl. Phys. B407 (1993) 356; Z. Fodor, J. Hein, K. Jansen, A. Jaster and I. Montvay, Nucl. Phys. B439 (1995) 147; K. Kajantie, M. Laine, K. Rummukainen and M.E. Shaposhnikov, Nucl. Phys. B466 (1996) 189; K. Jansen, Nucl. Phys. (Proc. Supl.) B47 (1996) 196; K. Rummukainen, Nucl. Phys. (Proc. Supl.) B53 (1997) 30

7. See e.g.: K. Kajantie, M. Laine, A. Rajantie, K. Rummukainen, M. Tsypin, hep-ph/9809435; and references therein 
8. G.R. Farrar and M.E. Shaposhnikov, Phys. Rev. Lett. 70 (1993) 2833 and (E): 71 (1993) 210; M.B. Gavela. P. Hernández, J. Orloff, O. Pène and C. Quimbay, Mod. Phys. Lett. 9 (1994) 795; Nucl. Phys. B430 (1994) 382; P. Huet and E. Sather, Phys. Rev. D51 (1995) 379

9. G.F. Giudice, Phys. Rev. D45 (1992) 3177; S. Myint, Phys. Lett. B287 (1992) 325

10. J.R. Espinosa, M. Quirós and F. Zwirner, Phys. Lett. B307 (1993) 106

11. A. Brignole, J.R. Espinosa, M. Quirós and F. Zwirner, Phys. Lett. B324 (1994) 181

12. M. Carena, M. Quirós and C.E.M. Wagner, Phys. Lett. B380 (1996) 81

13. D. Delepine, J.M. Gérard, R. González Felipe and J. Weyers, Phys. Lett. B386 (1996) 183

14. J.M. Moreno, D.H. Oaknin and M. Quirós, Nucl. Phys. B483 (1997) 267; Phys. Lett. B395 (1997) 234

15. J. Cline and K. Kainulainen, Nucl. Phys. B482 (1996) 73; Nucl. Phys. B510 (1998) 88-102

16. M. Laine, Nucl. Phys. B481 (1996) 43; M. Losada, Phys. Rev. D56 (1997) 2893; preprint [hep-ph/9612337]; G. Farrar and M. Losada, Phys. Lett. B406 (1997) 60

17. J.R. Espinosa, Nucl. Phys. B475 (1996) 273

18. B. de Carlos and J.R. Espinosa, Nucl. Phys. B503 (1997) 24

19. D. Bodeker, P. John, M. Laine and M.G. Schmidt, Nucl. Phys. B497 (1997) 387

20. M. Carena, M. Quiros and C.E.M. Wagner, Nucl. Phys. B524 (1998) 3, hep-ph/9710401

21. M. Dine, P. Huet, R. Singleton Jr. and L. Susskind, Phys. Lett. B257 (1991) 351; A. Cohen and A.E. Nelson, Phys. Lett. B297 (1992) 111; P. Huet and A.E. Nelson, Phys. Lett. B355 (1995) 229; Phys. Rev. D53 (1996)

22. M. Carena, M. Quiros, A. Riotto, I. Vilja and C.E.M. Wagner, Nucl. Phys. B503 (1997) 387 hep-ph/9702409

23. J. Cline, M. Joyce and K. Kainulainen, Phys. Lett. B417 (1998) 79

24. T. Multamaki, I. Vilja, Phys. Lett. B411 (1997) 301

25. A. Riotto, Int. J. Mod. Phys. D7 (1998) 815

26. M. Worah, Phys. Rev. D56 (1997) 2010

27. M. Carena and C.E.M. Wagner, preprint FERMILAB-PUB-97-095-T, hep-ph/9704347, in 'Perspectives on Higgs Physics II', ed. G. Kane, World Scientific, Singapore, 1997

28. H. Davoudiasl, K. Rajagopal and E. Westphal, Nucl. Phys. B515 (1998) 384 
29. Y. Brihaye and J. Kunz Phys. Rev. D48 (1993) 3884

30. J.M. Moreno, M. Quirós and M. Seco, Nucl. Phys. B526 (1998) 489

31. J. Cline and G.D. Moore, Phys. Rev. Lett. 81 (1998) 3317

32. P. John, e-print hep-ph/9810499

33. M. Laine and K. Rummukainen, Phys. Rev. Lett. 80 (1998) 5259; Nucl. Phys. B535 (1998) 423

34. J. Moreno, M. Quirós and M. Seco, in preparation.

35. J.M. Cline, G.D. Moore, G. Servant, e-print hep-ph/9902220

36. J.M. Cline, in these proceedings, e-print hep-ph/9902328 